\title{
On the Field Dependence of Random Walks in the Presence of Random Fields
}

\author{
A. Bunde, ${ }^{1,2}$ S. Havlin, ${ }^{1,2}$ H. E. Roman, ${ }^{3}$ G. Schildt, ${ }^{1}$ and H. E. Stanley ${ }^{1}$
}

\author{
Received November 24, 1987
}

\begin{abstract}
Numerical simulations and scaling arguments are used to study the field dependence of a random walk in a one-dimensional system with a bias field on each site. The bias is taken randomly with equal probability to be $+E$ or $-E$. The probability density $\bar{P}(x, t)$ is found to scale asymptotically as

$$
\left\{[A(E)]^{\beta / 2} / \mathrm{ln}^{2} t\right\} \exp \left(-\left\{x[A(E)]^{\beta / 2} / \mathrm{ln}^{2} t\right\}^{x}\right)
$$

with $A(E)=\ln [(1+E) /(1-E)], \beta=4.25$, and $\alpha=1.25$. The mean square displacement scales as $\left\langle x^{2}\right\rangle \sim[A(E)]^{-\beta} F\left[t A^{\beta}(E)\right]$, where $F(u) \sim \ln ^{4} u$ asymptotically.
\end{abstract}

KEY WORDS: Random walks; random fields; density distribution; fluctuations; anomalous diffusion.

The problem of anomalous transport in disordered media has received much interest in recent years (for reviews see, e.g., Refs. 1 and 2). The basic quantity characterizing diffusion is the probability density $P(x, t)$ for a random walker to be on a given substrate at site $x$ at time $t$ starting from $x=0$ at $t=0$. The study of the form of the configurational average of $P(x, t)$ as well as its fluctuations has received much attention. ${ }^{(3-10)}$

In this paper we study numerically and by scaling arguments the Sinai model, ${ }^{(11)}$ for which logarithmic anomalous diffusion has been found. Sinai

\footnotetext{
${ }^{1}$ Center for Polymer Studies and Department of Physics, Boston University, Bosiun, Massachusetts 02215.

${ }^{2}$ Department of Physics, Bar-Ilan University, Ramat-Gan, Israel.

${ }^{3}$ Fakultät für Physik, Universität Konstanz, D-775 Konstanz, Federal Republic of Germany and Institut für Theoretische Physik, Universität Hamburg, Jungiusstr. 9, D-2000 Hamburg 36, Federal Republic of Germany.
} 
studied a one-dimensional random walk in the presence of random fields and found for the mean-square displacement

$$
\left\langle x^{2}(t)\right\rangle \sim(\ln t)^{4}
$$

In this model the random walker experiences at each site a random bias field $E=p_{+}-p_{-}$. Here $p_{+}\left(p_{-}\right)$is the transition probability for a step to the right (left), with $p_{+}+p_{-}=1 ; p_{ \pm}$are taken from distributions where the mean value of $\ln \left(p_{+} / p_{-}\right)$is zero. Recently it has been argued that this type of random walk is related to random walks on random structures, such as random combs or the infinite percolation cluster at criticality under the influence of a constant bias field. ${ }^{(12,13)}$ In both systems, the mean-square displacement increases logarithmically with time and the fluctuations show multifractal behavior. ${ }^{(7,14)}$

The purpose of this work is to study the field dependence of $\left\langle x^{2}\right\rangle$ and of the mean (configurational averaged) distribution function for the case that the bias field can accept only two values $+E$ or $-E$ with equal

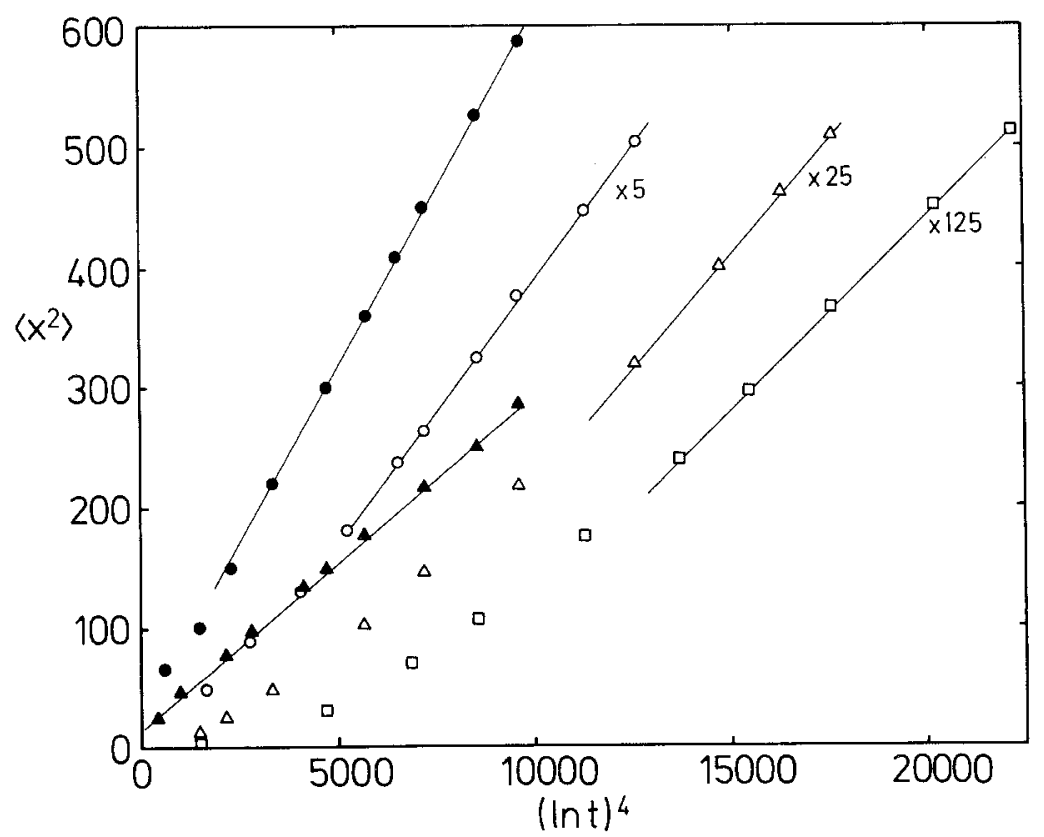

Fig. 1. Mean square displacement $\left\langle x^{2}\right\rangle$ versus $(\ln t)^{4}$ for $(\Delta) E=0.9,(\bullet) E=0.8,(\bigcirc)$ $E=0.6,(\triangle) E=0.4$, and $(\square) E=0.2$. The calculations were done on chains of up to 10000 sites and are based on averages over more than 200 configurations for each value of $E$. Note the different scale for different $E$ values. For $E=0.6,0.4$, and 0.2 the scale of the $y$ axis is multiplied with 5,25 , and 125 , respectively. 
probability. The case that $E$ is homogeneously distributed between 0 and 1 has been recently studied analytically by Kesten. ${ }^{(9)}$ For determining the mean distribution function $\bar{P}(x, t)$ and the mean-square displacement $\left\langle x^{2}(t)\right\rangle$ we used the exact enumeration method (see, e.g., Ref. 2). First we generated a linear chain with random fields $( \pm|E|)$ associated to each site. Then we solved the master equation on this chain to obtain $P(x, t)$ and the second moment. Finally, we averaged over many configurations. Our results for $\left\langle x^{2}(t)\right\rangle$, for various field strengths $E$, are shown in Fig. 1, where $\left\langle x^{2}(t)\right\rangle$ is plotted as a function of $(\ln t)^{4}$. For large times, the data fall on the straight lines, as predicted by (1). The mean-square displacement decreases with increasing bias field. Also, the crossover time to the asymptotic logarithmic behavior decreases with the field. The reason for this behavior is that the walker can get stuck in those regions of the chain where fields of opposite directions point toward the same site; the stronger the field, the more pronounced is this behavior.

In order to describe the field dependence of $\left\langle x^{2}(t)\right\rangle$, we assume the scaling form

$$
\left\langle x^{2}(t)\right\rangle=A(E)^{-\beta} F\left\{t[A(E)]^{\beta}\right\}
$$

where $A(E)=\ln [(1+E) /(1-E)]$. This scaling form yields the two known limits for $\left\langle x^{2}(t)\right\rangle$. For $t \rightarrow 0$ we expect $\left\langle x^{2}(t)\right\rangle \sim t$, independent of $E$, and

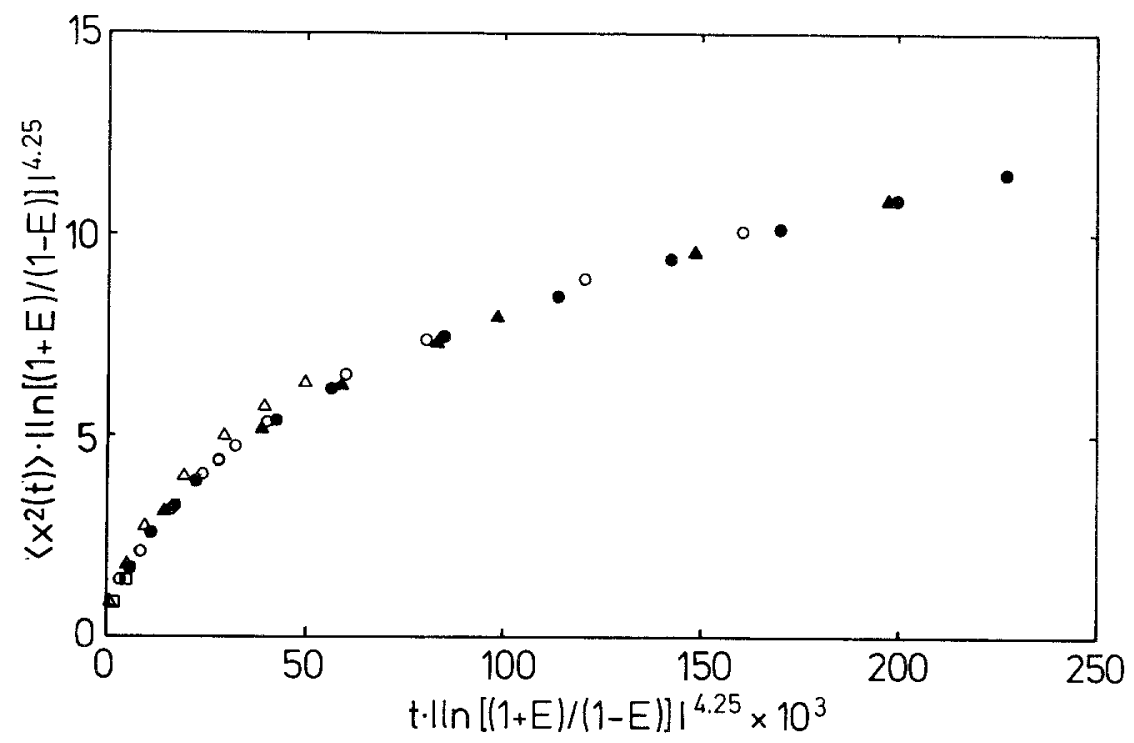

Fig. 2. Dependence of the scaled variable $\left\langle x^{2}\right\rangle A(E)^{\beta}$ on $t A(E)^{\beta}$, where $A(E)=$ $\ln [(1+E) /(1-E)]$ and $\beta=4.25$. The data collapse supports the scaling relation (2). The symbols correspond to the $E$ values of Fig. 1 . 
thus we have $F(u) \sim u$ for $u \ll 1$. For $t \rightarrow \infty$ we have $\left\langle x^{2}(t)\right\rangle \sim(\ln t)^{4}$ and thus $F(u) \sim(\ln u)^{4}$ for $u \gg 1$. We determined the exponent $\beta$ by fitting our numerical data from Fig. 1 to the scaling form, Eq. (2). We find that the best data collapse occurs for $\beta=4.25$ (see Fig. 2). Following the approach of Ref. 12 [Eq. (3)], we expect that $A(E)$ and $\ln t$ have the same exponent, i.e., $\beta=4$. The slightly higher value obtained here might be due to corrections to scaling which can be neglected only asymptotically.

Next we consider the mean density distribution $\bar{P}(x, t)$. A simple scaling form for $\bar{P}(x, t)$ which is consistent with (1) and (2) is

$$
\bar{P}(x, t)=\left[\left\langle x^{2}(t)\right\rangle\right]^{-1 / 2} G\left(x /\left\langle x^{2}(t)\right\rangle^{1 / 2}\right)
$$

To test this scaling form, in Fig. 3 we plot $\bar{P}(x, t)\left\langle x^{2}(t)\right\rangle^{1 / 2}$ as a function of

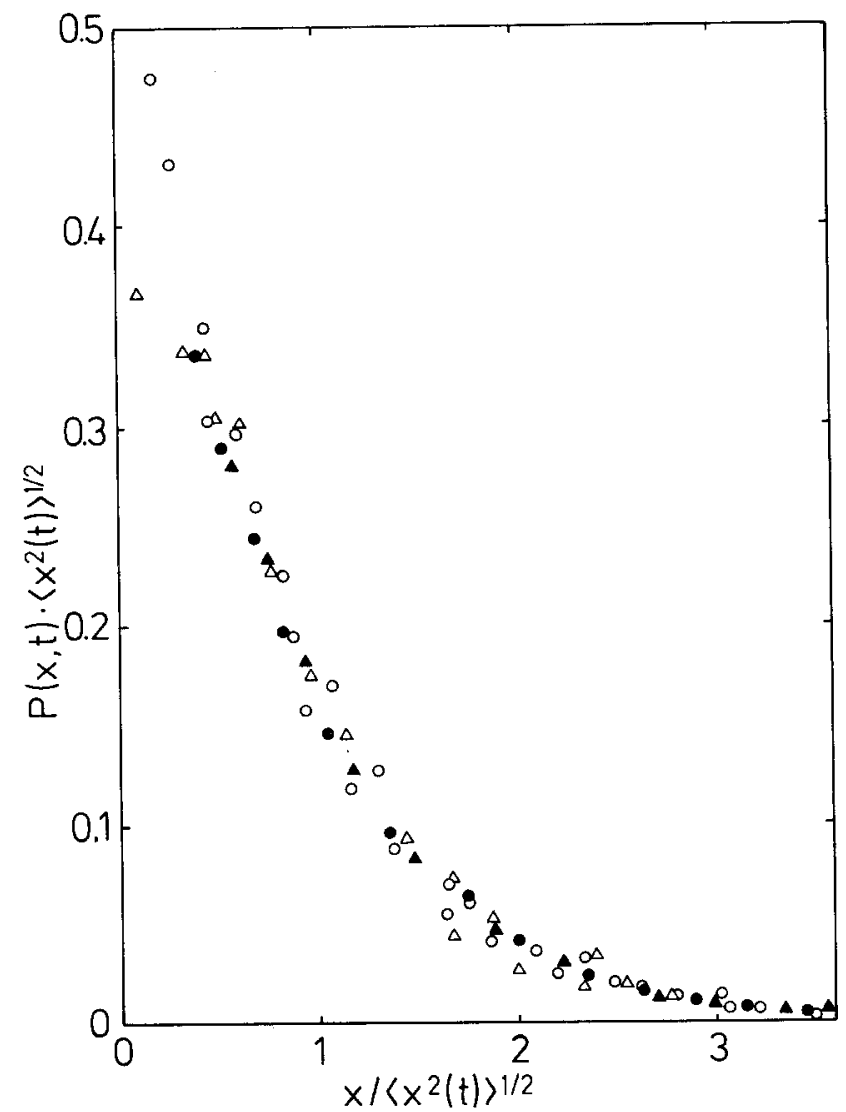

Fig. 3. The scaled mean density probability $\bar{P}(x, t)\left(\left\langle x^{2}\right\rangle\right)^{1 / 2}$ versus $x /\left(\left\langle x^{2}\right\rangle\right)^{1 / 2}$ for several values of $E$. The symbols correspond to the $E$ values of Fig. 1. The data collapse supports the scaling relation (3) for not too small $x$ values, $\left.x /\left(\left\langle x^{2}\right\rangle\right)^{1 / 2}\right\rangle 1 / 2$. 
$x /\left\langle x^{2}\right\rangle^{1 / 2}$ for several values of $E$. We find a good data collapse for large values of $x$ and $t$, indicating the validity range of the scaling. In general, the analytic form of $G(u)$ can be very complicated. As is convenient in the treatment of anomalous diffusion (see, e.g., Ref. 2), we assume that $G(u)$ approaches an exponential function for large $u$, i.e., $G(u) \sim \exp \left(-u^{\alpha}\right)$. To determine the exponent $\alpha$, we plot $\ln [\bar{P}(x, t) / \bar{P}(0, t)]$ as a function of $x$ in Fig. 4, for $E=0.8$ and two time values in the asymptotic region where $\left\langle x^{2}(t)\right\rangle \sim(\ln t)^{4}$. The curves are parallel straight lines with slope $\alpha=$ $1.25 \pm 0.05$. This value of $\alpha$ may be compared with the result $\alpha=1$ suggested by Nauenberg ${ }^{(8)}$ and proven analytically by Kesten ${ }^{(9)}$ for the case of homogeneously distributed fields. The difference can be due to the discreteness ( $\delta$-function distribution) of the bias field considered here, which might change the asymptotic behavior of $\bar{P}(x, t)$.

It is not unusual in anomalous diffusion that transport exponents are changed when passing from homogeneous to discrete distributions. For example, random walks in continuous percolation systems such as the

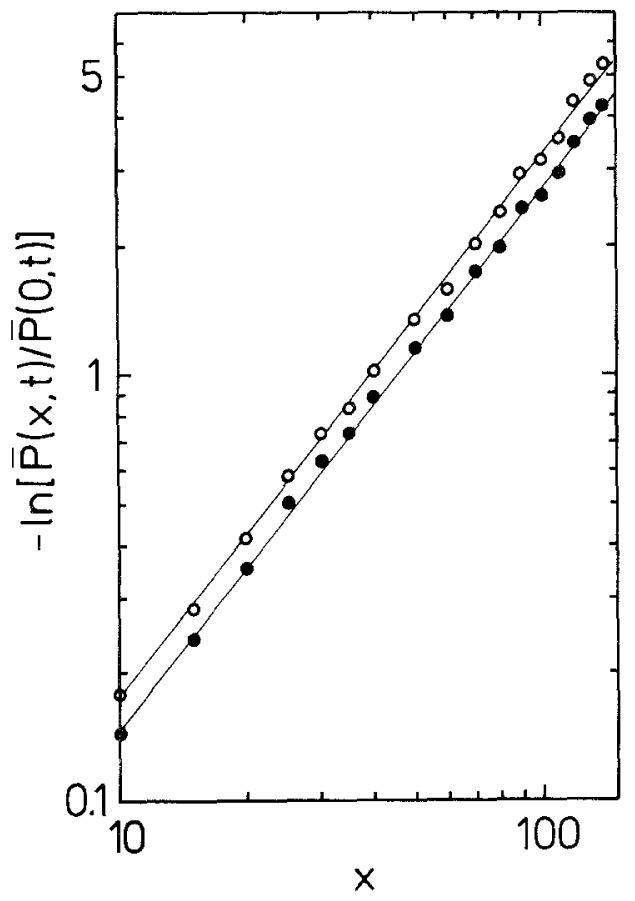

Fig. 4. Plot of $-\ln [\bar{P}(x, t) / \bar{P}(0, t)]$ versus $x$ for $E=0.8$ and $(O) t=10,000$ and (-) $t=20,000$. The slopes yields $\alpha=1.25$. The results are based on averages over 4000 configurations. 
Swiss cheese model are governed by diffusion exponents that differ from those of lattice percolation, though the static structures in both systems are in the same universality class. ${ }^{15,16)}$ The exponents are changed, furthermore, if in the continuous percolation system random walks with discrete fixed step length are considered. ${ }^{(17)}$

Another possibility for the discrepancy between our value of $\alpha=1.25$ and the value of $\alpha=1$ found by Kesten is that we did not yet reach the asymptotic regime where $\alpha=1$ can be observed. However, from the result of Kesten (Theorem 1.2 in Ref. 9) it follows that an effective exponent in the nonasymptotic regime would be smaller than 1 , in contrast to our finding.

\section{ACKNOWLEDGMENTS}

This work has been supported by Minerva and the Deutsche Forschungsgemeinschaft.

\section{REFERENCES}

1. J. W. Haus and K. W. Kehr, Phys. Rep. 150:263 (1987).

2. S. Havlin and D. Ben-Avraham, Adv. Phys. 36:695 (1987).

3. J. R. Banavar and J. Willemsen, Phys. Rev. B 30:6778 (1984).

4. B. O'Shaughnessy and I. Procaccia, Phys. Rev. Lett. 54:455 (1985); Phys. Rev. B 32:3073 (1985).

5. R. A. Guyer, Phys. Rev. A 29:2751 (1984).

6. S. Havlin, D. Movshovitz, B. L. Trus, and G. H. Weiss, J. Phys. A 18:L719 (1985).

7. H. Harder, S. Havlin, and A. Bunde, Phys. Rev. B 36:3874 (1987).

8. M. Nauenberg, J. Stat. Phys. 41:803 (1985).

9. H. Kesten, Physica 138A:299 (1986).

10. H. E. Roman, A. Bunde, and S. Havlin, Phys. Rev. A, in press.

11. Ya. Sinai, Theory Prob. Appl. 27:256 (1982).

12. S. Havlin, A. Bunde, Y. Glaser, and H. E. Stanley, Phys. Rev. A 34:3492 (1986).

13. A. Bunde and S. Havlin, Phil. Mag., in press.

14. A. Bunde, H. Harder, S. Havlin, and H. E. Roman, J. Phys. A 20:L865 (1987).

15. B. I. Halperin, S. Feng, and P. N. Sen, Phys. Rev. Lett. 54:2391 (1985).

16. A. Bunde, H. Harder, and S. Havlin, Phys. Rev. B 34:3540 (1986).

17. J. Petersen, H. E. Roman, A. Bunde, and W. Dieterich, Preprint. 